\title{
JSP Special Issue on Statistical Theory of Biological Evolution
}

\author{
Kavita Jain $^{1}$ - Luca Peliti ${ }^{2}$
}

Published online: 10 May 2018

(C) Springer Science+Business Media, LLC, part of Springer Nature 2018

In an influential essay, Dobzhansky famously stated that "nothing in biology makes sense except in the light of evolution". Indeed evolutionary processes shape diversity at all levels of biological organization, ranging from cellular to species and community level. In recent years, new methods and approaches have allowed to gain insight in evolutionary processes in real time via long-term evolution experiments or by high-resolution lineage tracking. Theoretical works using techniques borrowed from statistical physics and probability theory have helped greatly to understand the intricate process of evolution quantitatively. The objective of this special issue of Journal of Statistical Physics is to provide a view of some of the advances made in the field and of the current status of some outstanding and challenging problems.

A biological population evolves under the action of basic processes, viz., selection, mutation, migration and random genetic drift (stochastic fluctuations arising due to sampling) and its dynamics are described by high-dimensional partial differential equations that are, in general, not amenable to analysis. Constable and McKane show that by eliminating the fast degrees of freedom - an idea familiar to statistical physicists - the long-time behavior of an evolving population may be captured by simpler low-dimensional models. Pigolotti et al. take advantage of insights and techniques from non-equilibrium statistical physics in two spatial dimensions to gain a quantitative understanding of biodiversity measures such as the well-known phenomenological scaling laws between the number of species and the area of an ecosystem. Tikhonov and Monasson analyze the stability of complex ecosystems by a statistical physics approach to MacArthur's model of resource competition, and suggest that the intuition provided by a scalar notion of fitness may be misleading. Relationships between phenomena in statistical physics and evolutionary biology are further explored in the contri-

Luca Peliti

luca@peliti.org

Kavita Jain

jain@jncasr.ac.in

1 Theoretical Sciences Unit, Jawaharlal Nehru Centre for Advanced Scientific Research, Bangalore 560064, India

2 Santa Marinella Research Institute, 00058 Santa Marinella, Italy 
bution by Smerlak who discusses analogy between domain growth in physical systems and fitness growth in evolving biological populations.

Although adaptation is quite common, the adaptive process by which beneficial mutations that are initially rare spread in a population is still poorly understood. Different aspects of this challenging problem are discussed by several contributors in this issue. LeClair and Wahl review investigations on the effect of population bottleneck - that occur, for example, when a small fraction of microbial population is passaged to found a new colony-on the growth or loss of population fitness. The question of speed of adaptation which has recently been a subject of intense research is addressed by Danino, Kessler and Shnerb when the fitness fluctuates in time due to environmental variation. In cancer, certain mutants present in the primary tumor can develop drug resistance and their identification could help in better disease management. To achieve this goal, Rabadan et al. propose a model to separate the mutations of interest from noise in sequencing data.

Genealogical trees encode the information about the ancestry of the present population and have been used in the last three decades to infer useful information about the evolutionary process. The simplest continuous-time model known as the Kingman coalescent assumes neutral selection and finite variance in the offspring number, and current research has focused on relaxing these assumptions. In this issue, Baake and Wakolbinger review ancestral processes corresponding to a class of prototypical mutation-selection models, while Eldon and Freund present some new results for the genealogical trees for a population characterized by high variance in fecundity (such as certain marine organisms).

The metaphor of the fitness landscape introduced by Sewall Wright is at the same time appealing and problematic. Weinreich et al. investigate the effects that higher-order epistasis (the direct interaction of more than two loci) has on the topography of fitness landscape, suggesting new directions of theoretical and experimental work. Hwang et al. study fitness landscapes modeled by the celebrated Kauffman $N K$ model in the limit of large number of loci, by the methods of the statistical mechanics of disordered systems. They identify different universality classes determining the growth rate of local fitness maxima as a function of the number of loci.

The evolution of behavior is often tackled by means of evolutionary game theory. Arenzon and Canova revisit the Prisoner's Dilemma (PD) game by taking into account screening mechanisms, which tend to reduce the amount of interactions within a population. They exhibit the existence of a percolation-like threshold which separates a regime with a nonvanishing prevalence of loners to one in which the behavior reverts to the usual PD game. Perry et al. analyze the collective action problem for the case when individuals can not only contribute to the production of collective goods, but also punish free-riders. They contrast the dynamics of collective action in egalitarian and hierarchical groups. Their models predict the emergence of a division of labor in which more powerful individuals specialize in punishment while less powerful individuals mostly contribute to the production of collective goods.

This collection of works represents a fine sample of the current activity in evolutionary theory, and shows the relevance that methods inspired by statistical physics have acquired in this field. We hope that this special issue would spur further activity in the field and contribute towards a better understanding of the complex process of biological evolution. 